\title{
Independent delta/theta rhythms in the human hippocampus and entorhinal cortex
}

\author{
Florian Mormann ${ }^{1,2, *}$, Hannes Osterhage ${ }^{1,3}$, Ralph G. Andrzejak ${ }^{4}$, Bernd Weber ${ }^{1}$, \\ Guillén Fernández $^{5}$, Juergen Fell ${ }^{1}$, Christian E. Elger ${ }^{1}$ and Klaus Lehnertz ${ }^{1,3}$
}

1. Department of Epileptology, University of Bonn, Bonn, Germany

2. Division of Biology, California Institute of Technology, Pasadena, CA, USA

3. Helmholtz-Institute for Radiation and Nuclear Physics, University of Bonn, Bonn, Germany

4. Department of Information and Communication Technologies, University Pompeu Fabra, Barcelona, Spain

5. F.C. Donders Center for Cognitive Neuroimaging and Department of Neurology, Radboud University Nijmegen, Nijmegen,

The Netherlands

Edited by: Hans-Jochen Heinze, University of Magdeburg, Germany

Otto-von-Guericke-University, Germany

Reviewed by: Rodrigo Quian Quiroga, University of Leicester, UK

Christoph S. Herrmann, Otto-von-Guericke-University, Germany

Theta oscillations in the medial temporal lobe (MTL) of mammals are involved in various functions such as spatial navigation, sensorimotor integration, and cognitive processing. While the theta rhythm was originally assumed to originate in the medial septum, more recent studies suggest autonomous theta generation in the MTL. Although coherence between entorhinal and hippocampal theta activity has been found to influence memory formation, it remains unclear whether these two structures can generate theta independently. In this study we analyzed intracranial electroencephalographic (EEG) recordings from 22 patients with unilateral hippocampal sclerosis undergoing presurgical evaluation prior to resection of the epileptic focus. Using a wavelet-based, frequency-band-specific measure of phase synchronization, we quantified synchrony between 10 different recording sites along the longitudinal axis of the hippocampal formation in the non-epileptic brain hemisphere. We compared EEG synchrony between adjacent recording sites (i) within the entorhinal cortex, (ii) within the hippocampus, and (iii) between the hippocampus and entorhinal cortex. We observed a significant interregional gap in synchrony for the delta and theta band, indicating the existence of independent delta/theta rhythms in different subregions of the human MTL. The interaction of these rhythms could represent the temporal basis for the information processing required for mnemonic encoding and retrieval.

Keywords: medial temporal lobe, intracranial EEG, oscillations, synchronization, wavelet, phase precession

\section{INTRODUCTION}

Brain oscillations in the medial temporal lobe (MTL) of higher vertebrates have been a focus of neuroscientific research over the past decades. Particular emphasis has been placed on the role of theta oscillations that have been associated with a variety of cognitive functions in animals and humans, among these sensorimotor integration, spatial navigation, and mnemonic processing (cf. Buzsáki, 2005). Mediotempora theta oscillations have been found to act as a timing mechanism for neural activity (e.g., Jacobs et al., 2007; O'Keefe and Recce, 1993; Siapas et al., 2005; Skaggs et al., 1996) and to modulate higher frequency activity in the gamma range both in rodents (Bragin et al., 1995;

*Correspondence: Florian Mormann, Division of Biology, California Institute of Technology, MS 216-76, 1200 E California Boulevard, Pasadena, CA 91125, USA. e-mail: fmormann@ yahoo.de

Received: 25 January 2008; paper pending published: 08 April 2008; accepted: 06 May 2008; published online: 15 May 2008

Citation: Front. Hum. Neurosci. (2008) 2: 3. doi: 10.3389/neuro.09.003.2008

Copyright (๑) 2008 Mormann, Osterhage, Andrzejak, Weber, Fernández, Fell, Elger and Lehnertz. This is an open-access article subject to an exclusive license agreement between the authors and the Frontiers Research Foundation, which permits unrestricted use, distribution, and reproduction in any medium, provided the original authors and source are credited.
Chrobak and Buzsáki, 1998) and humans (Canolty et al., 2006; Demiralp et al., 2007; Mormann et al., 2005). Furthermore theta rhythmicity has been related to cognitive functions such as working memory (Jensen and Lisman, 1998) and to separate dynamics of associative synaptic encoding and retrieval (Hasselmo et al., 2002). One of the seminal discoveries in the context of mediotemporal theta oscillations was the phenomenon of phase precession (0'Keefe and Recce, 1993; Skaggs et al., 1996). By firing periodically but with a slight frequency mismatch relative to their local field theta activity, place cells in the rodent hippocampus create a temporal code - defined by the phase relationship to the concurrent theta cycle - that can represent the position of an animal more accurately and dissociated from the place cell's firing rate (Huxter et al., 2003).

The mechanisms responsible for the generation of theta activity in the MTL are complex and not entirely understood (see e.g., Buzsáki, 2002). One original hypothesis is that cholinergic excitation from the medial septum and the diagonal band of Broca activates inhibitory interneurons, which in turn induce rhythmic IPSPs on the soma of pyramidal cells (Petsche et al., 1962). More recent data from rodent hippocampal slice preparations and in anesthetized rats indicate that theta activity can also be generated intrinsically in the CA1 region of the hippocampus (Gillies et al., 2002; Rotstein et al., 2005) and the parasubiculum (Glasgow and Chapman, 2007). Analyses of hippocampal 
recordings in awake rats have provided evidence for at least two hippocampal theta rhythms of which only one is mediated by inputs from the entorhinal cortex (Kocsis et al., 1999). Although lesion studies in rodents have shown a strong influence of entorhinal inputs on hippocampal theta activity (Bragin et al., 1995; Buzsaki et al., 1983 Kamondi et al., 1998), to our knowledge, no direct comparison between entorhinal and hippocampal theta activity has been reported in animals or humans to date.

In this study we analyzed intracranial electroencephalographic (EEG) recordings from patients with pharmaco-refractory unilateral epilepsy of the MTL who were undergoing invasive diagnostics prior to resective surgery of the seizure-generating area. During this procedure patients were implanted with intracranial depth electrodes to localize the focus of seizure onset. We take advantage of the fact that in our patient group these electrodes were positioned along the longitudinal axis of the MTL (Spencer, 1987) with contacts both in the hippocampus and in the entorhinal cortex, thereby allowing us to determine the level of synchrony within and between these structures from electrode contacts that are spaced apart at a constant distance, which would not be possible for depth electrodes implanted orthogonally to this axis (Olivier et al., 1987). In addition we apply strictest criteria to ascertain unilaterality of the epileptic process based on histopathological evaluation of the resected tissue and assessment of post-operative seizure control. Within this framework, we address the question whether the interplay between the human hippocampus and entorhinal cortex is governed by a common (global) theta rhythm or whether both regions exhibit essentially independent theta rhythms.

\section{MATERIALS AND METHODS Subjects}

The patients analyzed in this study were selected from an original group of 40 patients with strictly unilateral pharmacoresistant MTL epilepsy who underwent invasive presurgical evaluation between 1993 and 2000 and for whom at least $15 \mathrm{~min}$ of seizure-free recordings were archived. Of these 40 patients, only 22 patients ( 15 women; mean age $32.1 \pm 5.5$ years) passed our inclusion criterion of having two or more electrode contacts in the entorhinal cortex adjacent to two or more contacts in the hippocampus in the hemisphere contralateral to the epileptic focus (see next section). In seven patients seizures originated exclusively from the right MTL; in 15 patients exclusively from the left MTL. After resection of the epileptic MTL, all patients remained seizurefree (follow-up at least 6 months, mean: 3.3 years). In all patients, preimplantational MRI showed unilateral hippocampal sclerosis, which was confirmed by histopathological examination of the resected tissue. All additional non-invasive diagnostic findings were concordant with the MRI diagnosis. No additional pathology was indicated. At the time of clinical evaluation of these patients, intracranial electrode implantation was deemed necessary to confirm diagnosis prior to resective surgery (cf. Diehl and Luders, 2000). The EEG study was approved by the medical ethics committee of the University of Bonn. Each patient gave written informed consent.

\section{Depth electrodes}

Bilateral depth electrodes, each equipped with 10 cylindrical contacts (length: $2.5 \mathrm{~mm}$, intercontact distance: $4 \mathrm{~mm}$ ), were inserted along the longitudinal axis of the hippocampal formation using a computed tomography-based stereotactic insertion technique (Van Roost et al., 1998), with the anterior contacts located ventral to the amygdala in the anterior parahippocampal gyrus, which is covered by peri- and entorhinal cortex, and the posterior contacts located within the hippocampus (Figure 1).

The location of electrode contacts was ascertained by MRI in each patient. MRI scans were acquired in sagittal, adjusted coronal (perpendicular to the longitudinal axis of the hippocampus), and axial (parallel to

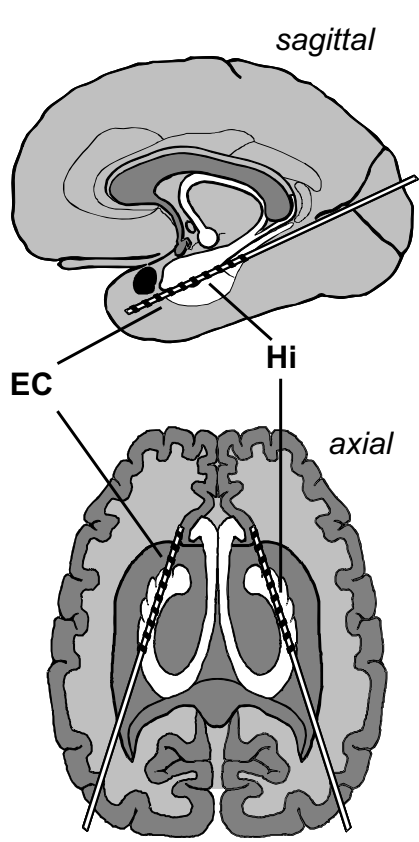

Figure 1. Electrode implantation scheme. Longitudinal depth electrodes are placed with the anterior contacts located in the entorhinal cortex (EC) and the posterior contacts located in the hippocampus (Hi).

this axis) planes, typically in $2 \mathrm{~mm}$ sliced $\mathrm{T} 1$ - and T2-weighted sequences as well as $3 \mathrm{~mm}$ sliced FLAIR sequences. Anatomical boundaries were ascertained according to Insausti et al. (1998). Among others, we used the collateral sulcus, hippocampal fissure, and sulcus semiannularis as landmarks to delineate the entorhinal cortex from neighboring structures. As detailed above, only patients with at least two clearly entorhinal contacts adjacent to at least two clearly hippocampal contacts were included in the study.

\section{Intracranial EEG recordings}

EEG recordings were acquired at a sampling frequency of $173.61 \mathrm{~Hz}$ using a 12-bit analog to digital (A/D) converter, band-pass filtered from 0.5 to $85 \mathrm{~Hz}$ (12 dB/oct.) and with a $50-\mathrm{Hz}$ notch filter, and referenced against the common average of the non-focal recording contacts. Mean recording time per patient was $130 \mathrm{~min}$ (range 15-420 min, typically recorded over several days). All recordings were performed during the seizure-free interval while patients were awake and at rest with eyes opened. Only EEG recordings from the MTL contralateral to the zone of seizure origin were analyzed in the present study to reduce poorly controllable effects introduced by the epileptic process (Grunwald et al., 1995).

\section{Data analysis}

We divided the continuous EEG signals into consecutive segments $s(t)$ of $23.6 \mathrm{~s}$ (4096 data points) and calculated the mean phase coherence $R$ as a measure of the degree of phase synchrony (Mormann et al., 2000) from all artifact-free segments (using an automated artifact detection algorithm to exclude segments with signal saturation and steep transients) for every possible combination of electrode contacts $x$ and $y$ within the MTL of the hemisphere contralateral to the epileptic focus, resulting in a symmetrical 10 by 10 synchronization matrix $\left\{R_{x, y}\right\}$

$$
R_{x, y}=\left|\frac{1}{N} \sum_{k=1}^{N} \exp i\left[\phi_{x}(k \cdot \Delta t)-\phi_{y}(k \cdot \Delta t)\right]\right|
$$

with $N=4096$ and $\Delta t$ denoting the sampling interval. 
The phase variables $\phi_{x, y}$ for different frequency bands were calculated using a wavelet-based approach (Torrence and Compo, 1998), with the center frequency and bandwidth of a continuous Morlet wavelet adapted to match conventional EEG bands, i.e., the $\delta(0.5-4 \mathrm{~Hz}), \theta(4-8 \mathrm{~Hz})$, $\alpha(8-13 \mathrm{~Hz}), \beta_{1}(13-20 \mathrm{~Hz}), \beta_{2}(20-30 \mathrm{~Hz})$, and $\gamma_{1}(30-49 \mathrm{~Hz})$ band, at full width half maximum (Osterhage et al., 2007):

$\phi^{\mathrm{band}}(t)=\arctan \frac{\operatorname{Im} W^{\text {band }}(t)}{\operatorname{Re} W^{\text {band }}(t)}$

where $W^{\text {band }}(t)$ denotes the wavelet coefficient

$W^{\text {band }}(t)=\int_{-\infty}^{+\infty} \mu^{\text {band }}(t-\tau) s(\tau) d \tau$

of a Morlet wavelet

$\mu^{\text {band }}(t)=\left(e^{i \omega_{0}^{\text {band } t}}-e^{-\frac{\left(\omega_{0}^{\text {band }} \sigma^{\text {band }}\right)^{2}}{2}}\right) e^{-\frac{1}{2}\left(\frac{t}{\sigma^{\text {band }}}\right)^{2}}$

with

$\sigma^{\text {band }}=\frac{2 \pi \sqrt{\ln 2}}{\Delta \omega^{\text {band }}}$

Each frequency band is thus defined by its center frequency $\omega_{0}^{\text {band }}$ and bandwidth $\Delta \omega^{\text {band }}$, e.g., for the $\theta$-band $(4-8 \mathrm{~Hz})$ we use $\omega_{0}^{\text {band }}=6 \mathrm{~Hz}$ and $\Delta \omega^{\theta}=4 \mathrm{~Hz}$.
Note that the measure $R$ is completely independent of the amplitudes of the time series as well as any constant phase lag between them. Furthermore this measure has been shown to reliably reflect relative changes in synchrony even in the strong presence of noise (Mormann et al., 2000). Since the variance over time was very low for the obtained synchrony profiles $(<0.01$ on average for different contact combinations, frequency bands, and patients, cf. Bullock et al., 1995b), matrices were subsequently averaged over time, resulting in a single synchronization matrix per patient and EEG band (cf. Figure 2).

\section{Statistical analysis}

From the 10 electrode contacts analyzed in each patient, we identified a series of four consecutive contacts such that the two anterior contacts of this series were located in the entorhinal cortex and the two posterior contacts in the anterior hippocampus. This series of four consecutive contacts thus contained three pairs of adjacent contacts, one within the entorhinal cortex, one extending across the border between the entorhinal cortex and hippocampus, and one within the hippocampus.

For the three pairs of adjacent contacts we then extracted from the temporally averaged synchronization matrices of each patient the mean degree of synchrony within the entorhinal cortex, between entorhinal cortex and hippocampus, and within the hippocampus, respectively (Figure 2A), and compared these mean values across patients using a nonparametric test (two-sided Wilcoxon signed-rank test). This procedure ensured that the compared synchrony values were calculated from pairs of contacts that were all spaced at the same distance.

A)



B)

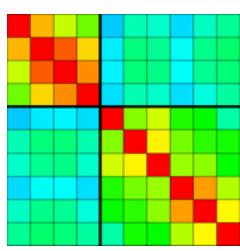

$\delta$ - band

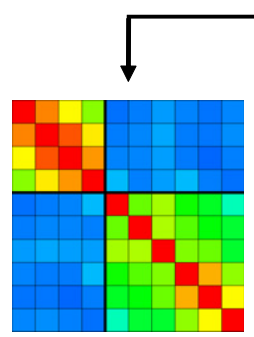

$\theta$ - band

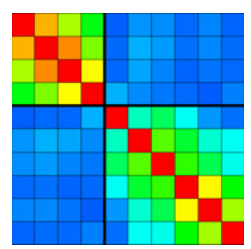

$\alpha-$ band

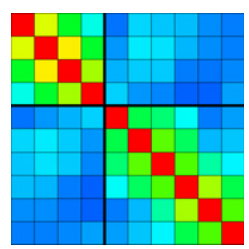

$\beta_{1}$ - band

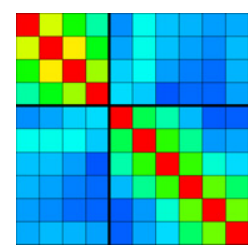

$\beta_{2}$ - band

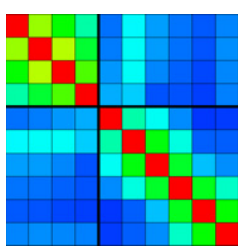

$\gamma_{1}$ - band

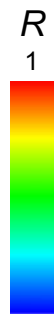

0

Figure 2. Exemplary results. (A) Exemplary traces of bandpass-filtered theta activity from two entorhinal (EC, channels 3 and 4) and two hippocampal (Hi, channels 5 and 6) recording sites. The average degree of synchrony $R$ between these recording sites over time is color-coded and displayed in the synchronization matrix (upper right) for this EEG frequency band. In this schematic, only the three matrix entries for which the subsequent group statistics was performed are filled. (B) Synchronization matrices from the same patient for different EEG bands, including the theta matrix from (A), now with all entries filled. 
A gap in inter-regional synchrony was considered statistically significant if for any given frequency band the inter-regional (entorhinalhippocampal) synchrony was lower than both intra-regional synchronies (i.e., within entorhinal cortex and within hippocampus) at the $p=0.05$ significance level of the two-sided Wilcoxon test. Assuming the onesided probabilities for this hypothesis (EC-Hi lower than EC-EC and $\mathrm{EC}-\mathrm{Hi}$ lower than $\mathrm{Hi}-\mathrm{Hi}$ ) to be independent and including a Bonferroni correction for testing multiple (six) frequency bands, one obtains an effective significance value for a gap in synchrony defined in this way as $p=0.025 \times 0.025 \times 6<0.004$. However, since one of the paired samples (EC-Hi) is used in both tests, the assumption of independent probabilities is not valid. We therefore used Monte Carlo simulations based on samples of pseudorandom numbers to estimate the actual empirical $p$-value of our combined criterion (including the Bonferroni correction) as $p<0.024$.

A gap in inter-regional synchrony in a given frequency band in turn was interpreted as evidence for the presence of independent rhythms in this frequency band.

\section{RESULTS}

Figure 2A shows examples of bandpass-filtered theta activity from a patient in whom the anterior four contacts were located in the entorhinal cortex, while the posterior six contacts were located in the hippocampus. The two entorhinal traces (channels 3 and 4) exhibit an oscillatory pattern that differs from the hippocampal traces (channels 5 and 6 ), particularly in the first and last third of the displayed traces. Accordingly, the average degree of synchrony between the adjacent entorhinal and hippocampal channels $(R=0.18)$ is substantially lower than the degree of synchrony between the two entorhinal channels $(R=0.85)$ and the two hippocampal channels $(R=0.60)$, respectively.

Figure 2B shows that for this patient all four entorhinal contacts exhibit relatively high mutual synchrony (upper left $4 \times 4$ quadrants) as do all six hippocampal contacts (lower right $6 \times 6$ quadrants). The level of synchrony between these two structures, i.e., between entorhinal and hippocampal contacts, is distinctly lower (upper right and lower left $4 \times 6$ regions). This difference between intra- and inter-regional synchrony is discernible in all frequency bands but is most pronounced for the theta band in this particular patient.

The synchronization matrices in Figure 2B illustrate how the degree of synchrony generally decreases with increasing distance between recordings sites, even within the same anatomical region, which is in accordance with earlier findings (Bullock et al., 1995a). To compare intra- and inter-regional synchrony, we therefore restricted the statistical analysis to the two most posterior entorhinal and the two most anterior hippocampal contacts, yielding from anterior to posterior three overlapping pairs of adjacent recording sites within the entorhinal cortex (EC-EC), on both sides of the entorhinal-hippocampal border (EC-Hi), and within the hippocampus $(\mathrm{Hi}-\mathrm{Hi}$ ), respectively (cf. Figure $2 \mathrm{~A}$ ). Synchrony values were thus calculated from contacts spaced at a constant distance.

Pairwise comparison of averaged intra- and inter-regional synchronization values across patients showed in all frequency bands highest synchrony levels within the hippocampus, followed by high intra-entorhinal synchrony, and comparably low levels of synchrony between these two structures (Figure 3). This effect was most pronounced in the delta and theta band, where inter-regional synchrony was significantly lower than intra-regional synchrony in either the entorhinal cortex or the hippocampus. Levels of synchrony generally tended to decrease with increasing EEG band frequency.

\section{DISCUSSION}

We analyzed EEG recordings from the unaffected MTLs of a group of 22 patients with strictly unilateral epilepsy of the MTL as confirmed by postoperative seizure control. Evaluation of the degree of synchrony within the hippocampus, within the entorhinal cortex, and between these
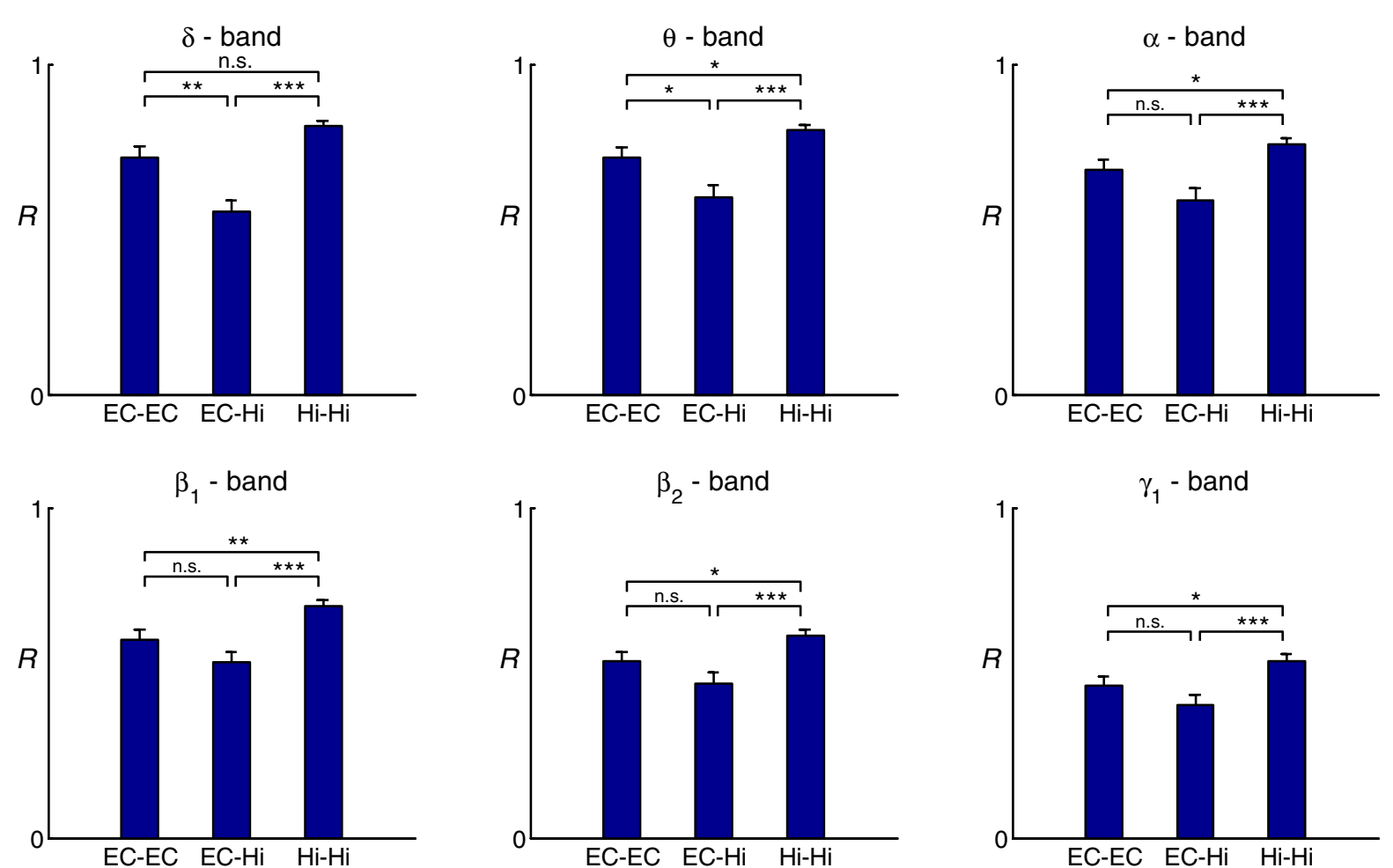

Figure 3. Group statistics for 22 patients. Comparison of intra- and inter-regional synchrony within and between the entorhinal cortex and hippocampus for different frequency bands. Bars depict mean levels of synchrony across patients within the entorhinal cortex (EC-EC), between entorhinal cortex and hippocampus (EC-Hi), and within the hippocampus (Hi-Hi), respectively, with error bars denoting the standard error. $n . S .=$ not significant, $*=p<0.05, * *=p<0.01$, $* * *=p<0.001$ (two-sided Wilcoxon test; uncorrected $p$-values). Note that a statistically significant inter-regional gap in synchrony as defined in the methods section is observed only for the delta and theta band.

4 
two structures revealed that entorhinal-hippocampal synchrony was lower than synchrony within either of these structures throughout different EEG bands, with this effect reaching statistical significance only in the delta and theta band. Our findings thus indicate the presence of independent delta/theta rhythms in the entorhinal cortex and hippocampus in humans. The presence of a significant interregional drop in synchrony not only in the theta band, but also (and even more pronounced) in the delta band deserves discussion, because delta and subdelta oscillations are usually found in neocortex and during slow-wave sleep or non-physiological conditions. Since the hippocampal theta oscillations observed in rodents are known to be shifted towards lower frequencies in higher animal species (Buzsáki, 2006), it is conceivable that the human analog of rodent MTL theta extends into the delta frequency range. Alternatively, a shift of the oscillatory activity towards lower frequencies in our subjects could reflect an indirect influence of the contralateral epileptic process.

While the overall degree of measured synchrony could in principle be biased towards higher values by effects of volume conduction and the use of a common average reference in the recording montage, neither of these factors can account for the distinct gap in synchrony observed between the two anatomical regions as they would, conversely, act to level out this effect. More precisely, different conductivities in the two regions could theoretically affect the amplitudes of a presumed global rhythm and even introduce a (potentially frequency-specific) phase shift. However, either of these effects would be constant over time and therefore not affect the measure for synchrony we used in this study, which is independent of the power, i.e., the amplitudes, in either of the two signals from which it is calculated, or any constant phase lag between them. Although a recent study assessing the impedance spectrum in monkey cortex showed impedance to be homogeneous, tangentially isotropic, and not frequency-dependent (Logothetis et al., 2007), this may not necessarily be the case for the hippocampus due to its laminar structure, which in theory could generate electrical fields along its longitudinal axis (Taylor and Dudek, 1984). While such an effect can not account for the observed inter-structural gap in synchrony, it could possibly be the reason for the higher level of intra-hippocampal synchrony compared to intra-entorhinal synchrony.

The observed gap in inter-structural synchrony implies the existence of independent delta/theta rhythms in the human MTL, produced by autonomous generators. Regarding the potential generating mechanisms of these rhythms, findings from rodent electrophysiology have provided multiple indications of local theta generators in different subregions of the hippocampus as well as in the entorhinal cortex (Acker et al., 2003; Alonso and Llinas, 1989; Giocomo et al., 2007). The interplay between these subregions, however, is complex and not entirely understood. Perhaps the most important findings in this context were derived from recordings in awake rats, where partial coherence analyses of hippocampal recordings provided evidence for at least two hippocampal theta generators in the hippocampus of which only one is mediated by inputs from the entorhinal cortex (Kocsis et al., 1999). In the light of this study, the two independent theta rhythms found in humans could thus be interpreted such that the entorhinal rhythm may represent intrinsic entorhinal rhythmicity mediated by sensory and other cortical inputs, while the hippocampal rhythm could reflect theta activity generated autonomously within the hippocampus itself, mediated by inputs from the medial septum and the diagonal band of Broca. We may speculate further that the two independent rhythms observed here might represent the two oscillators of slightly different frequencies postulated by the interference model of theta phase precession (O'Keefe and Recce, 1993). To actually test these hypotheses, however, simultaneous recordings of local field potentials and unit activity as well as an improved spatial sampling and more accurate subregional localization of the recording contacts would be necessary.

The idea of multiple autonomous theta generators in humans is in agreement with findings from intracranial recordings in patients with different types of focal epilepsy during a working memory task, which have indicated local mechanisms for the generation of cortical theta (Raghavachari et al., 2006), although no systematic comparison of MTL subregions was performed in this study.

The role of mediotemporal theta activity in memory processing has long been established by rodent studies showing that LTP in the hippocampus is optimally elicited with stimulation at theta frequency and that discrete lesions in the medial septum that abolish theta produce severe learning/ memory deficits (for an overview see e.g., Vertes and Kocsis, 1997 or Axmacher et al., 2006). A recent study using intracranial recordings from patients with temporal lobe epilepsy performing a word list-learning paradigm showed a significant increase in rhinal-hippocampal theta coherence for subsequently remembered versus forgotten words (Fell et al., 2003). Given our present findings, we can hypothesize that the independent theta rhythms found in the entorhinal cortex and hippocampus may need to be actively synchronized in order to facilitate the synaptic plasticity involved in memory encoding. This hypothesis is further backed by the fact that in the rodent hippocampus the theta phase at which a stimulus arrives determines the direction of plasticity, i.e., whether it results in long term potentiation or depression. This effect as been shown both for artificially induced theta activity in vivo (Pavlides et al., 1988) and in vitro (Huerta and Lisman, 1995) as well as in behaving animals (Holscher et al., 1997; Hyman et al., 2003). In this context it is conceivable that synchronization of theta activity between the hippocampus and entorhinal cortex creates a slowly modulated facilitating state that provides the temporal basis for encoding or retrieval of separate items (Hasselmo et al., 2002; Jensen and Lisman, 1998; Rizzuto et al., 2006). Such a synchronization process could, e.g., be instantiated by a simultaneous phase reset of ongoing oscillatory activity in both regions, driven by sensory inputs (Givens, 1996; McCartney et al., 2004). In a recent study we demonstrated such a stimulus-induced theta phase reset in humans during a continuous word recognition paradigm. This reset occurred approximately $190 \mathrm{~ms}$ after stimulus onset and was observed simultaneously in the hippocampus and rhinal cortex (Mormann et al., 2005).

Future studies in humans using more localized recording techniques, e.g., via microwires (Fried et al., 1997), in combination with novel computerassisted localization techniques of electrode contacts (Ekstrom et al., 2008) may help to further elicit the generating mechanisms and interplay of these rhythms and their functional relevance for mnemonic processing at the single neuron level.

\section{CONFLICT OF INTEREST STATEMENT}

The authors declare that the research was conducted in the absence of any commercial or financial relationships that could be construed as a potential conflict of interest.

\section{ACKNOWLEDGEMENTS}

We thank Christof Koch, Costas Anastassiou, Gyorgy Buzsaki, and Eva Pastalkova for fruitful discussion. This work was supported by the Intramural Research Fund BONFOR of the University of Bonn and the $6^{\text {th }}$ Framework Programme of the European Commission (Marie Curie OIF 040445).

\section{REFERENCES}

Acker, C. D., Kopell, N., and White, J. A. (2003). Synchronization of strongly coupled excitatory neurons: relating network behavior to biophysics. J. Comput. Neurosci. $15,71-90$.

Alonso, A., and Llinas, R. R. (1989). Subthreshold Na+-dependent theta-like rhythmicity in stellate cells of entorhinal cortex layer II. Nature 342, 175-177.

Axmacher, N., Mormann, F., Fernández, G., Elger, C. E., and Fell, J. (2006). Memory formation by neuronal synchronization. Brain Res. Brain Res. Rev. 52, 170-182.

Bragin, A., Jandó, G., Nádasdy, Z., Hetke, J., Wise, K., and Buzsáki, G. (1995). Gamma $(40-100 \mathrm{~Hz})$ oscillation in the hippocampus of the behaving rat. J. Neurosci. 15, $47-60$.

Bullock, T. H., McClune, M. C., Achimowicz, J. Z., Iragui-Madoz, V. J., Duckrow, R. B. and Spencer, S. S. (1995a). EEG coherence has structure in the millimeter domain: subdural and hippocampal recordings from epileptic patients. Electroencephalogr. Clin. Neurophysiol. 95, 161-177. 
Bullock, T. H., McClune, M. C., Achimowicz, J. Z., Iragui-Madoz, V. J., Duckrow, R. B., and Spencer, S. S. (1995b). Temporal fluctuations in coherence of brain waves. Proc. Natl. Acad. Sci. USA 92, 11568-11572.

Buzsáki, G. (2002). Theta oscillations in the hippocampus. Neuron 33, 325-340.

Buzsáki, G. (2005). Theta rhythm of navigation: link between path integration and landmark navigation, episodic and semantic memory. Hippocampus 15, 827-840.

Buzsáki, G. (2006). Rhythms of the Brain. Oxford University Press, New York.

Buzsaki, G., Leung, L. W., and Vanderwolf, C. H. (1983). Cellular bases of hippocampal EEG in the behaving rat. Brain Res. 287, 139-171.

Canolty, R. T., Edwards, E., Dalal, S. S., Soltani, M., Nagarajan, S. S., Kirsch, H. E., Berger, M. S., Barbaro, N. M., and Knight, R. T. (2006). High gamma power is phaselocked to theta oscillations in human neocortex. Science 313, 1626-1628.

Chrobak, J. J., and Buzsáki, G. (1998). Gamma oscillations in the entorhinal cortex of the freely behaving rat. J. Neurosci. 18, 388-398.

Demiralp, T., Bayraktaroglu, Z., Lenz, D., Junge, S., Busch, N. A., Maess, B., Ergen, M., and Herrmann, C. S. (2007). Gamma amplitudes are coupled to theta phase in human EEG during visual perception. Int. J. Psychophysiol. 64, 24-30.

Diehl, B., and Luders, H. 0. (2000). Temporal lobe epilepsy: when are invasive recordings needed? Epilepsia 41, S61-S74.

Ekstrom, A., Suthana, N., Behnke, E., Salamon, N., Bookheimer, S., and Fried, I. (2008) High-resolution depth electrode localization and imaging in patients with pharmacologically intractable epilepsy. J. Neurosurg. 108, 812-815.

Fell, J., Klaver, P., Elfadil, H., Schaller, C., Elger, C. E., and Fernández, G. (2003). Rhinalhippocampal theta coherence during declarative memory formation: interaction with gamma synchronization? Eur. J. Neurosci. 17, 1082-1088.

Fried, I., MacDonald, K. A., and Wilson, C. L. (1997). Single neuron activity in human hippocampus and amygdala during recognition of faces and objects. Neuron 18 753-765.

Gillies, M. J., Traub, R. D., LeBeau, F. E. N., Davies, C. H., Gloveli, T., Buhl, E. H., and Whittington, M. A. (2002). A model of atropine-resistant theta oscillations in rat hippocampal area CA1. J. Physiol. 543, 779-793.

Giocomo, L. M., Zilli, E. A., Fransen, E., and Hasselmo, M. E. (2007). Temporal frequency of subthreshold oscillations scales with entorhinal grid cell field spacing. Science 315, 1719-1722.

Givens, B. (1996). Stimulus-evoked resetting of the dentate theta rhythm: relation to working memory. Neuroreport 8, 159-163.

Glasgow, S. D., and Chapman, C. A. (2007). Local generation of theta-frequency EEG activity in the parasubiculum. J. Neurophysiol. 97, 3868-3879.

Grunwald, T., Elger, C. E., Lehnertz, K., Van Roost, D., and Heinze, H. J. (1995) Alterations of intrahippocampal cognitive potentials in temporal lobe epilepsy. Electroencephalogr. Clin. Neurophysiol. 95, 53-62.

Hasselmo, M. E., Bodelon, C., and Wyble, B. P. (2002). A proposed function for hippocampal theta rhythm: separate phases of encoding and retrieval enhance reversal of prior learning. Neural Comput. 14, 793-817.

Holscher, C., Anwyl, R., and Rowan, M. J. (1997). Stimulation on the positive phase of hippocampal theta rhythm induces long-term potentiation that can be depotentiated by stimulation on the negative phase in area CA1 in vivo. J. Neurosci. 17, 6470-6477.

Huerta, P. T., and Lisman, J. E. (1995). Bidirectional synaptic plasticity induced by a single burst during cholinergic theta oscillation in CA1 in vitro. Neuron 15, 1053-1063.

Huxter, J., Burgess, N., and O'Keefe, J. (2003). Independent rate and temporal coding in hippocampal pyramidal cells. Nature 425, 828-832.

Hyman, J. M., Wyble, B. P., Goyal, V., Rossi, C. A., and Hasselmo, M. E. (2003). Stimulation in hippocampal region CA1 in behaving rats yields long-term potentiation when delivered to the peak of theta and long-term depression when delivered to the trough. J. Neurosci. 23, 11725-11731.

Insausti, R., Juottonen, K., Soininen, H., Insausti, A. M., Partanen, K., Vainio, P., Laakso, M. P., and Pitkanen, A. (1998). MR volumetric analysis of the human entorhinal, perirhinal, and temporopolar cortices. AJNR. Am. J. Neuroradiol. 19, 659-671.

Jacobs, J., Kahana, M. J., Ekstrom, A. D., and Fried, I. (2007). Brain oscillations control timing of single-neuron activity in humans. J. Neurosci. 27, 3839-3844.
Jensen, O., and Lisman, J. E. (1998). An oscillatory short-term memory buffer model can account for data on the Sternberg task. J. Neurosci. 18, 10688-10699.

Kamondi, A., Acsády, L., Wang, X. J., and Buzsáki, G. (1998). Theta oscillations in somata and dendrites of hippocampal pyramidal cells in vivo: activity-dependent phaseprecession of action potentials. Hippocampus 8, 244-261.

Kocsis, B., Bragin, A., and Buzsáki, G. (1999). Interdependence of multiple theta generators in the hippocampus: a partial coherence analysis. J. Neurosci. 19, 6200-6212.

Logothetis, N. K., Kayser, C., and Oeltermann, A. (2007). In vivo measurement of cortical impedance spectrum in monkeys: implications for signal propagation. Neuron 55 , 809-823.

McCartney, H., Johnson, A. D., Weil, Z. M., and Givens, B. (2004). Theta reset produces optimal conditions for long-term potentiation. Hippocampus 14, 684-687.

Mormann, F., Fell, J., Axmacher, N., Weber, B., Lehnertz, K., Elger, C. E., and Fernández, G. (2005). Phase/amplitude reset and theta-gamma interaction in the human medial temporal lobe during a continuous word recognition memory task. Hippocampus $15,890-900$.

Mormann, F., Lehnertz, K., David, P., and Elger, C. E. (2000). Mean phase coherence as a measure for phase synchronization and its application to the EEG of epilepsy patients. Physica D 144, 358-369.

O'Keefe, J., and Recce, M. L. (1993). Phase relationship between hippocampal place units and the EEG theta rhythm. Hippocampus 3, 317-330.

Olivier, A., Marchand, E., Peters, T., and Tyler, J. (1987). Depth electrode implantation at the Montreal Neurological Institute and Hospital. In Surgical Treatment of the Epilepsies, J. Engel Jr, ed. (New York, NY, Raven Press), pp. 595-601.

Osterhage, H., Mormann, F., Wagner, T., and Lehnertz, K. (2007). Measuring the directionality of coupling: phase versus state space dynamics and application to EEG time series. Int. J. Neural Syst. 17, 139-148.

Pavlides, C., Greenstein, Y. J., Grudman, M., and Winson, J. (1988). Long-term potentiation in the dentate gyrus is induced preferentially on the positive phase of theta-rhythm. Brain Res. 439, 383-387.

Petsche, H., Stumpf, C., and Gogolak, G. (1962). The significance of the rabbit's septum as a relay station between the midbrain and the hippocampus. I. The control of hippocampus arousal activity by the septum cells. Electroencephalogr. Clin. Neurophysiol. 14, 202-211.

Raghavachari, S., Lisman, J. E., Tully, M., Madsen, J. R., Bromfield, E. B., and Kahana, M. J. (2006). Theta oscillations in human cortex during a working-memory task: evidence for local generators. J. Neurophysiol. 95, 1630-1638.

Rizzuto, D. S., Madsen, J. R., Bromfield, E. B., Schulze-Bonhage, A., and Kahana, M. J. (2006). Human neocortical oscillations exhibit theta phase differences between encoding and retrieval. Neuroimage 31, 1352-1358.

Rotstein, H. G., Pervouchine, D. D., Acker, C. D., Gillies, M. J., White, J. A., Buhl, E. H., Whittington, M. A., and Kopell, N. (2005). Slow and fast inhibition and an H-current interact to create a theta rhythm in a model of CA1 interneuron network. J. Neurophysiol. 94, 1509-1518.

Siapas, A. G., Lubenov, E. V., and Wilson, M. A. (2005). Prefrontal phase locking to hippocampal theta oscillations. Neuron 46, 141-151.

Skaggs, W. E., McNaughton, B. L., Wilson, M. A., and Barnes, C. A. (1996). Theta phase precession in hippocampal neuronal populations and the compression of temporal sequences. Hippocampus 6, 149-172.

Spencer, D. D. (1987). Depth electrode implantation at Yale University. In: Surgical Treatment of the Epilepsies, J. Engel Jr, ed. (New York, NY, Raven Press), pp. 603-608.

Taylor, C. P., and Dudek, F. E. (1984). Excitation of hippocampal pyramidal cells by an electrical field effect. J. Neurophysiol. 52, 126-142.

Torrence, C., and Compo, G. P. (1998). A practical guide to wavelet analysis. Bull. Am. Meteorol. Soc. 79, 61-78.

Van Roost, D., Solymosi, L., Schramm, J., van Oosterwyck, B., and Elger, C. E. (1998). Depth electrode implantation in the length axis of the hippocampus for the presurgical evaluation of medial temporal lobe epilepsy: a computed tomography-based stereotactic insertion technique and its accuracy. Neurosurgery 43, 819-826.

Vertes, R. P., and Kocsis, B. (1997). Brainstem-diencephalo-septohippocampal systems controlling the theta rhythm of the hippocampus. Neuroscience 81, 893-926. 